\title{
Effect of incorrect sound velocity on synthetic aperture sonar resolution
}

\author{
Xia $\mathrm{Ji}^{*}$, Lisheng Zhou, and Weihua Cong \\ Science and Technology on Sonar Laboratory Sciences, Hangzhou Applied Acoustics Research Institute, Hangzhou, China
}

\begin{abstract}
Synthetic aperture sonar (SAS) is an imaging technique to produce centimeter resolution over hundreds-of-meter range on the sea floor, by constructing a virtual aperture whose length automatically adjusts itself for a given focusing range. SAS is near-field acoustic imaging, and this implies that the sound velocity should be accurately estimated for well focused imaging. Otherwise there will be image quality loss. However, sound velocity in the ocean varies with space and time, and there might also be measuring error of CTD (Conductivity, Temperature, and Depth) sensor, so sound velocity error has become one of the limiting factors to improve SAS resolution further. To characterize the effect of sound velocity error quantificationally, the practice SAS resolution is mode as the convolution of ideal seafloor reflectivity function and a phase error function in frequency domain, where the phase error is caused by incorrect sound velocity. Then the SAS resolution parameterized is calculated as a function of the sound velocity measuring error, or sound velocity gradient. It is shown that SAS azimuthally (along track) resolution loss, caused by sound velocity measurement error, increases linearly with detection range. Meanwhile the loss caused by sound velocity gradient increases squarely. It is simulated by considering the synthetic aperture data collection for a particular pixel, and results show that the point scatter response will defocus when the sound velocity measuring error is up to $1 \%$ at $200 \mathrm{~m}$ range, or the sound velocity changes up to $2 \%$ over a typical gradient at $200 \mathrm{~m}$ range, and be worse at a longer range. Furthermore, we demonstrate the influence of sound velocity errors on SAS imagery using a sea trial data and real CTD measurements at South China Sea. We evaluate the degradation in image quality with respect to sound velocity errors by using two plastic balls and a variable seafloor scene, and results also support the accuracy of theoretical conclusions above.
\end{abstract}

\section{Introduction}

Synthetic aperture sonar (SAS) is an innovative and high technology in the 21 st ocean century. Because of its key role in achieving long-distance high-resolution in low frequency detection, SAS becomes a powerful tool for underwater imaging and has broad application prospects in military and national economic fields[1-2]. Because of combining signals coherently of a dummy long array in near-field, SAS requires accurate sound velocity. However, sound velocity in ocean varies with space and time, so sound velocity error has become one of the main limiting factors to further improve synthetic aperture sonar resolution, especially the low frequency and long detection range SAS. Researching the effect of incorrect sound velocity on synthetic aperture sonar resolution not only deepen the understanding but also helps to system design and sound velocity correction [3-5].

The basic principle of synthetic aperture sonar is introduced in this paper, and the effect of phase error on SAS resolution. The formula of SAS azimuth (along track) resolution is developed when sound velocity is measured incorrectly, and the effect of sound velocity gradient on SAS is analyzed too. It is calculated that extended value of SAS beam width, causes by sound velocity measurement error, increased linearly with detection range. Meanwhile the value caused by sound velocity gradient increases squarely. Simulation results show accuracy in conclusion.

\section{Azimuth resolution of SAS}

SAS is emerging as an imaging technology that can provide centimeter resolution over hundreds-of-meter range. Fig. 1 is the geometric model of SAS, where $r$ is the range value, $x$ is the azimuth value, $P(0, r)$ is a target in strip map.

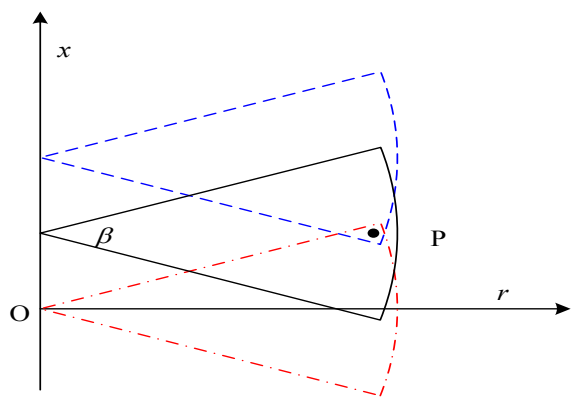

Fig. 1. Geometric model of SAS.

"Corresponding author: 1x060721@,163.com 
SAS transmit a linear frequency mapping (LFM) signal

$$
s(t)=e^{j\left(2 \pi f_{t} t+\rho t^{2}\right)} 0 \leq t \leq T_{p}
$$

where $\mathrm{t}$ is time, $\mathrm{t}=0$ at the moment of $\mathrm{P}$ target exposured by the transmited beam, $\mathrm{B}$ is the bandwidth, $T_{p}$ is pulse width, signal chip rate $\rho=\pi B / T_{p}$. Signal received by hydrophone is

$$
\begin{aligned}
e(t) & =K s(t-\tau) \\
& =K(x, r) e^{j\left(2 \pi f_{l}(t-\tau)+\rho(t-\tau)^{2}\right)}
\end{aligned}
$$

Symbol $\tau$ is the delay time of echo, $\tau \approx 2 r / c^{+}+\left(v t-x_{0}\right)^{2} / r c$, and $v$ is the sonar platform velocity.

When Formula 2 can further be simplified to

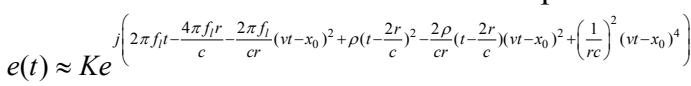

$$
\begin{aligned}
& \left.\approx K e^{j\left(2 \pi f_{t}\left(t-\frac{2 r}{c}\right)+\rho\left(t-\frac{2 r}{c}\right)^{2}\right)} e^{-j\left(\frac{2 \pi f v^{2}}{c r}\left(t-x_{0} / v\right)^{2}\right.}\right) \\
& \approx K s\left(t-\frac{2 r}{c}\right) e^{-j\left(\frac{2 \pi f v^{2}\left(t-x_{0} / v\right)^{2}}{c r}\right)} \quad\left(v t-x_{0}\right)<<r
\end{aligned}
$$

It can be found that there is an addition phase function because of sonar platform moving.

$$
\phi(t)=e^{-j \frac{2 \pi f v^{2}\left(t-x_{0} /\right)^{2}}{c r}}\left|t-\frac{x_{0}}{v}\right| \leq T_{S A}
$$

The physical meaning of addition phase function is doppler changing history where $T_{S A}$ is time period of synthetic aperture sonar. Synthetic aperture sonar takes uniform linear motion as a basic assumption. The addition phase function is a LFM signal, so the matched filter normalized output of $\phi(t)$ is

$$
|R(t)|=\left|\frac{\sin \left(\frac{4 \pi v^{2}}{\lambda r} T_{S A}\left(t-x_{0} / v\right)\right)}{\frac{4 \pi v^{2}}{\lambda r} T_{S A} t\left(t-x_{0} / v\right)}\right|
$$

where $T_{S A}$ is $r \lambda / D v$. Formula 5 can further be simplified to

$$
|R(t)|=\left|\frac{\sin \left(\frac{4 \pi v}{D}\left(t-x_{0} / v\right)\right)}{\frac{4 \pi v}{D}\left(t-x_{0} / v\right)}\right|
$$

In Formula (6), D is the physical array length. So the $-3 \mathrm{~dB}$ beam width is

$$
\Delta t_{S A}=\frac{D}{2 v}
$$

then by multiplying $\Delta t_{S A}$ with sonar platform velocity, we can get SAS azimuth resolution

$$
\Delta x=v \Delta t_{S A}=D / 2
$$

It can be concluded that SAS azimuth resolution has nothing to do with signal frequency and detection range, but constant for half the physical array length.

In practice, due to fluctuation of medium, motion error and channel consensus, there are always unwanted phase errors, which lead to the mismatch with SAS signal processing model. Suppose the unwanted phase errors to be $g(t)$, doppler changing history function is

$$
\widetilde{\phi}(t)=\operatorname{rect}\left(\frac{1}{T_{S A}}\left(t-x_{0} / v\right)\right) g(t) e^{-j \frac{2 \pi f_{v^{2}}\left(t-x_{0} / v\right)^{2}}{c r}}
$$

to analyze easily, we change $\widetilde{\phi}(t)$ into frequency domain

$$
\widetilde{\Phi}\left(k_{x}\right)=\Phi\left(k_{x}\right) \otimes G\left(k_{x}\right)
$$

where $\Phi\left(k_{x}\right)=\operatorname{rect}\left(k_{x} D / 4 \pi\right)$ is the frequency spectrum of $\phi(t)$, and $G\left(k_{x}\right)$ is the frequency spectrum of $g(x)$. It can be concluded from formula 10 that $\Phi\left(k_{x}\right)$ is the ideal spectrum. So matched filter normalized output is

$$
|R(t)| \approx\left|\frac{\sin \left(\frac{4 \pi v^{2}}{\lambda r}\left(T_{S A}-1 / B_{x}\right)\left(t-x_{0} / v\right)\right)}{\frac{4 \pi v^{2}}{\lambda r}\left(T_{S A}-1 / B_{x}\right)\left(t-x_{0} / v\right)}\right|
$$

where B is the bandwidth of $G\left(k_{x}\right)$, So when there are phase errors, SAS azimuth resolution is

$$
\Delta \widetilde{x} \approx \frac{D}{2}+\frac{\lambda r}{4 \pi} B_{k_{x}}
$$

and $B_{k_{x}}=2 \pi B_{x} / v$ means azimuth wave number.

It can be found that the effect of phase error is related to doppler band width, target range, and signal frequency.

\section{The effect of velocity measure error}

Synthetic aperture sonar measures water conductive, ocean temperature and depth by CTD, and then the sound velocity was calculated by empircal formula. However ocean environment is time-space changing. So there is always measure error, and there is gradient changing too.

When there is velocity measure error, it will lead to relative errors in the geometry

$$
\begin{aligned}
g(t) & =\operatorname{rect}\left(\frac{t-\frac{x_{0}}{v}}{T_{S A}}\right) e^{j \Delta k \sqrt{v^{2}\left(t x^{x_{0}} /\right)^{2}+r^{2}}} \\
& \approx \operatorname{rect}\left(\frac{\left.t-\frac{x_{0} / v}{T_{S A}}\right) e^{j \Delta k r} e^{j \Delta k \frac{v^{2}\left(t-x_{0} / v\right)^{2}}{2 r}}}{}\right.
\end{aligned}
$$

where $\Delta c$ is velocity measure error, and $\Delta k=k \Delta c / c$. It can be found from formula (10) that phase error caused by velocity measure error can be divided into two parts. The first part is time independent, and would not worsen the azimuth resolution, but position accuracy. The second part changes the chirp rate of doppler, which means azimuth beam broadening.

Azimuth frequency spectrum of $g(t)$ can be written as

$$
G\left(k_{x}\right)=\int r e c t\left(\frac{t-x_{0} / v}{T_{S A}}\right) e^{j \Delta k \frac{v^{2}\left(t-x_{0} / v\right)^{2}}{2 r}-j k_{x} v t} d t
$$


where both windows function $\operatorname{rect}\left(\frac{1}{T_{S A}}\left(t-x_{0} / v\right)\right)$ and phase function $\phi(t)=\frac{j \Delta k v^{2}}{2 r}\left(t-x_{0} / v\right)^{2}-j k_{x} v t$ vary with time slowly, so according to stationary principle

$$
\int f(t) e^{j \phi\left(t_{0}\right)} d t=\sqrt{\frac{j 2 \pi}{\phi^{\prime \prime}\left(t_{0}\right)}} f\left(t_{0}\right) e^{j \phi\left(t_{0}\right)}
$$

where $t_{0}$ is a pole of $\phi(t)$. It can be derived that $t_{0}=\frac{k_{x} r-2 \Delta k x_{0}}{2 \Delta k v}$ from $\left.\phi^{\prime}(t)\right|_{t=t_{0}}=0$. So $G\left(k_{x}\right)$ can be written as

$$
G\left(k_{x}\right)=\sqrt{\frac{j \pi r}{\Delta k}} \operatorname{rect}\left(\frac{k_{x} r}{2 \Delta k v T_{S A}}\right) e^{j \frac{k_{x}^{2} r}{4 \Delta k}-k_{x} x_{0}}
$$

Inserting formula (12) in (16), we get the extension of SAS azimuth resolution

$$
\begin{aligned}
\Delta \widetilde{x} & =\frac{D}{2}+\left|\frac{\Delta k}{2 k}\right| v T_{S A} \text { where }\left|\frac{\Delta c}{c}\right| \leq \frac{\pi r}{2 L_{S A}^{2}} \\
& =\frac{D}{2}+\left|\frac{\Delta c}{c}\right| \frac{\lambda}{D} r
\end{aligned}
$$

where LSA is the length of the synthetic aperture, $L_{S A}=v T_{S A}$.

It is proved that extension azimuth resolution, caused by velocity measure error, increased linearly with detection range.

\section{The effect of sound velocity gradient}

In small-scale ocean environment, the sound velocity varies with depth mainly. According to Leroy empirical formula, when glancing angle is constant, the sound velocity changes linearly with detection range $r$

$$
\widetilde{c} \approx c+\alpha r
$$

where $\alpha$ is the sound velocity gradient. Here we introduce a parameter $c^{\prime}$ that is called equivalent sound velocity, and the parameter must satisfy

$$
2 \int_{0}^{r} \frac{d y}{c+\alpha y}=\frac{2 r}{c^{\prime}}
$$

According to taylor expansion $\ln (1+x) \approx x-x^{2} / 2$, formula (19) can further be simplify to

$$
c^{\prime}=\alpha r \ln \left(1+\frac{\alpha r}{c}\right)^{-1}=c+\frac{\alpha r}{2}
$$

Inserting (12) in (16), we get the extension of SAS azimuth resolution

$$
\Delta \widetilde{x} \approx \frac{D}{2}+\left|\frac{\alpha r}{c}\right| L_{S A} \approx \frac{D}{2}+\left|\frac{\alpha}{2 c}\right| \frac{\lambda}{D} r^{2}
$$

where $r$ is the height of sonar platform form ocean bottom.

It is proved that extension azimuth resolution, caused by sound velocity gradient, has relation with sonar platform height and glancing angle.

\section{Simulation and sea trial data analysis}

It is concluded from theoretical analysis that both sound velocity measuring error and sound velocity gradient can worsen SAS azimuth resolution. In this chapter we will research the effect of incorrect sound velocity on SAS resolution using a simulation and sea trial data at South China Sea.

Firstly, an SAS system is simulated with frequency $50 \mathrm{kHz}$, physical aperture of length $1.28 \mathrm{~m}$ with 8 elements. When there is sound velocity measure error up to $0.5 \%$ and $1 \%$, image of a point target at different range is showed in Fig.2, and the azimuth of points target with different sound velocity measure errors is showed in Fig.3.

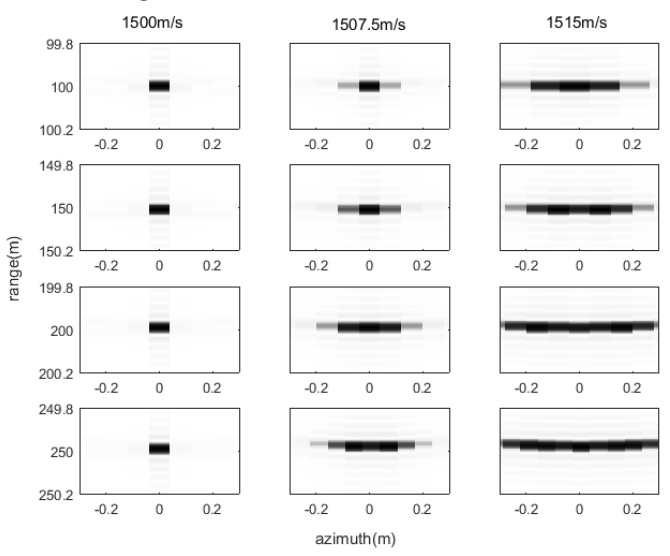

Fig. 2. Point target images with $0.5 \%$ or $1 \%$ sound velocity measure error.

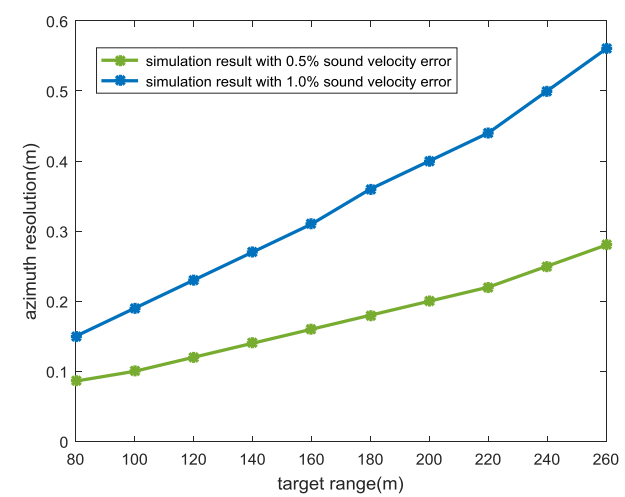

Fig. 3. The azimuth of target point with different sound velocity measure errors.

As shown in Fig.2 and Fig.3, the target point images are defocusing because of sound velocity measure errors, the azimuth resolution extend more than $0.36 \mathrm{~m}$ from theoretical resolution $0.08 \mathrm{~m}$ for targets at more than $200 \mathrm{~m}$ range when sound velocity measure errors are up to $1 \%$, which can not be accepted. And the azimuth resolution worsens linearly with detection range, and this also proved formula (17).

Keeping SAS system parameter constant, Supposed there a linear and negative sound velocity gradient with $7.5 \mathrm{~m} / \mathrm{s}$ every $100 \mathrm{~m}$, or $15 \mathrm{~m} / \mathrm{s}$ every $100 \mathrm{~m}$, image of point targets at different ranges are showed in Fig. 4 and Fig.5. 


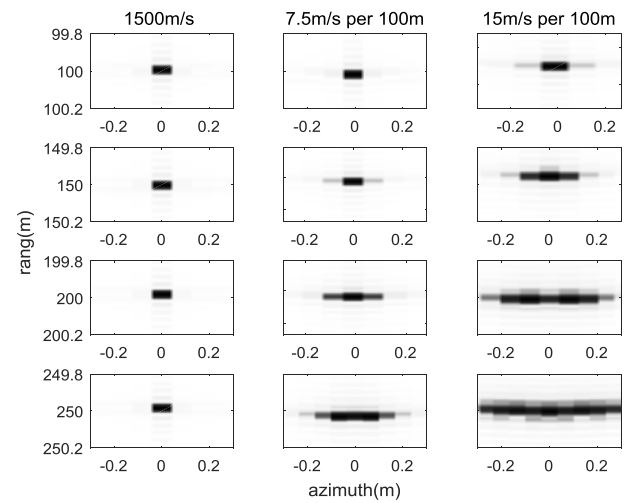

Fig. 4. Point target images with linear negative sound velocity gradient.

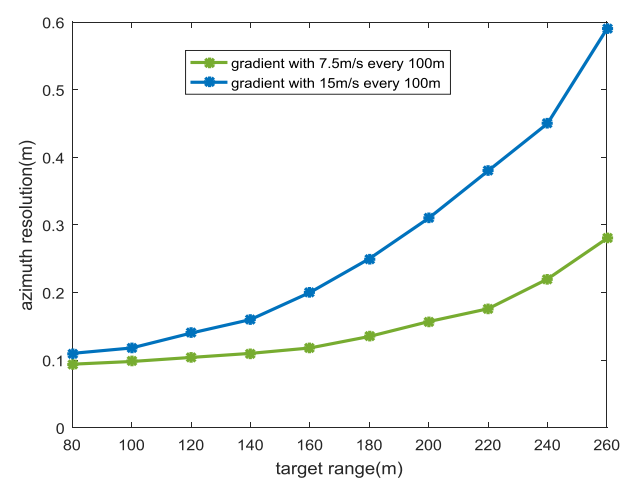

Fig. 5. The azimuth of target point with different sound velocity measure errors.

As shown in Fig.4 and Fig.5, azimuth of the point target, at $200 \mathrm{~m}$ range, extends to $0.31 \mathrm{~m}$ from the theoretical resolution $0.08 \mathrm{~m}$ when there is a linear sound velocity gradient. Generally speaking SAS azimuth resolution worsens with detection range, and beam width caused by sound velocity gradient increases squarely with range.

We analyze sea trial data collected by a SAS system in 2016 at South China Sea. And sound velocity gradient is obtained by the on-board measurement device, which is shown in Fig.6. And there are two suspended plastic balls in the scene. The azimuth of small plastic balls can be used to judge on the effect of incorrect sound velocity on synthetic aperture sonar resolution.

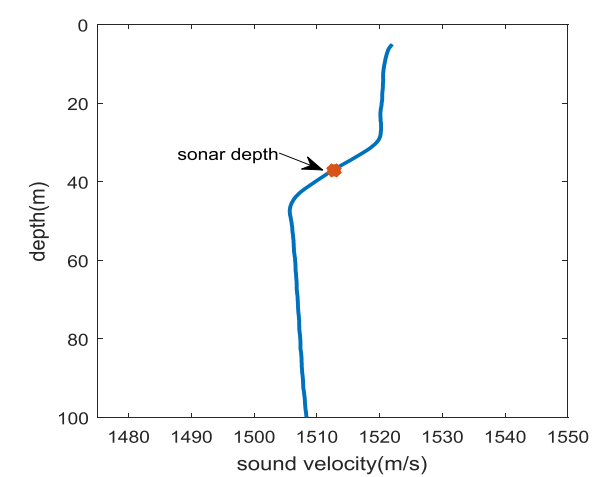

Fig. 6. Sound velocity gradient in the sea area.

According to formula (19), the optimum sound speed has been estimated to be about $1509 \mathrm{~m} / \mathrm{s}$, Fig7 is the well focused SAS image of the 2016 South China Sea data. Fig.8(a) and (b) use the correct sound speed, and are therefore well focused. An error of $9 \mathrm{~m} / \mathrm{s}$ has been induced during the reconstruction process of the SAS image shown in (c) and (d). The quality of the depicted in (c) and (d) suffers severely from the errors, and the ball at $236 \mathrm{~m}$ defocused more serious than the closer one. Neglecting the sound velocity variations with depth, we take the sound speed $1514 \mathrm{~m} / \mathrm{s}$ obtained by sonar platform, and SAS images of the two suspended balls are shown in (e) and (f). Results show that the obtained sound speed yields a defocus too, with $1 \#$ ball fuzzier than $2 \#$ ball.

Compared to the results in fig8, it is illustrated that defocus would become apparent if there is an incorrect sound speed measurement, and sound velocity gradient should not be ignored too, especially the low frequency and long detection range of SAS system.

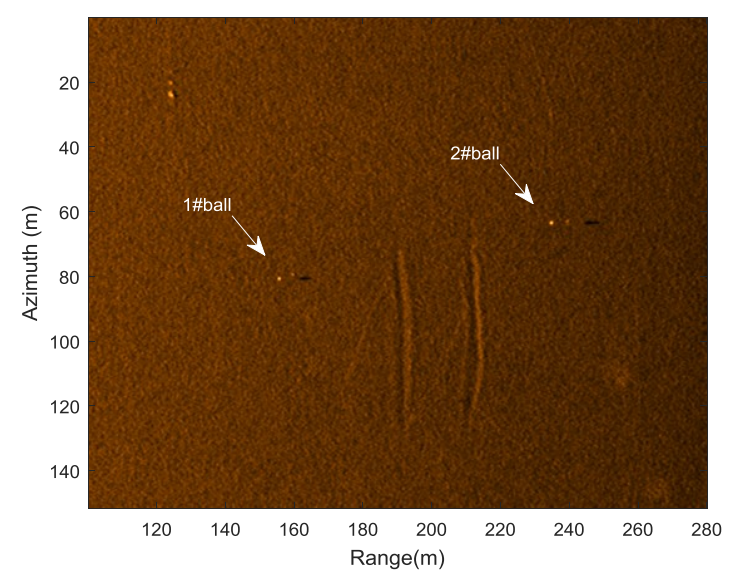

Fig. 7. Well focused SAS image of 2016 South China Sea data set.

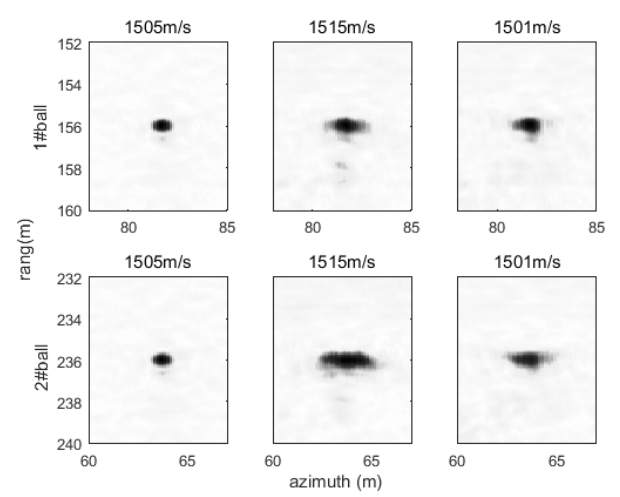

Fig. 8. SAS image of plastic balls, the optimum sound speed $1500 \mathrm{~m} / \mathrm{s}$ in (a) and (b), an incorrect sound speed measurement $1500 \mathrm{~m} / \mathrm{s}$ in (c) and (d), obtained sound speed $1514 \mathrm{~m} / \mathrm{s}$ by sonar platform in (e) and (f) are used.

\section{Conclusions}

We have concluded from theoretical analysis that both sound velocity measuring error and sound velocity gradient can worsen SAS azimuth resolution. Theoretical analysis result shows that SAS azimuthally resolution loss, caused by sound velocity measurement error, increases linearly with detection range, meanwhile and the loss caused by sound velocity gradient increases squarely. Simulation synthetic sonar data were used to demonstrate the influence of sound speed errors, variations of simulated PSF caused by incorrect sound 
velocity consistent with theoretical conclusion. Further, sea trial data were used to demonstrate the influence of sound speed errors on SAS imagery, result indicates that both incorrect sound speed measurement and sound velocity gradient should be accounted for.

\section{References}

1. M. P. Hayes and P. T. Gough, IEEE J. Ocean. Eng., 34, 207-224(2009)

2. R. E. Hansen and H. J. Callow, IEEE Transaction on geoscience and remote sensing, 49, 3677-3687(2011)

3. X. Ji, W. H. Cong, L. S. Zhou, IEEE/OES China ocean acoustics symposium 2016

4. B. B. Zhu, W. H. Cong, IEEE/OES China ocean acoustics symposium 2016

5. Z. J. Yue, T. Wang, IEEE/OES China ocean acoustics symposium 2016 